\title{
Réflexions sur la pertinence de la notion de contexte dans les études relatives aux activités informationnelles
}

On the Notion of Context in Research on Information Activities

\section{Céline Paganelli}

\section{Q OpenEdition}

Journals

Édition électronique

URL : https://journals.openedition.org/edc/6545

DOI : $10.4000 /$ edc. 6545

ISSN : 2101-0366

Éditeur

Université de Lille

Édition imprimée

Date de publication : 1 juin 2016

Pagination : 165-188

ISBN : 978-2-917562-15-4

ISSN : $1270-6841$

\section{Référence électronique}

Céline Paganelli, «Réflexions sur la pertinence de la notion de contexte dans les études relatives aux activités informationnelles », Études de communication [En ligne], 46 | 2016, mis en ligne le 01 juin 2018, consulté le 08 décembre 2022. URL : http://journals.openedition.org/edc/6545 ; DOI : https:// doi.org/10.4000/edc.6545 
Réflexions sur la pertinence de la notion de contexte dans les études relatives aux activités informationnelles

On the Notion of Context in Research on Information Activities 
L'article propose une étude du contexte dans les recherches relatives aux activités informationnelles. Une revue de la littérature montre que cette notion, souvent floue et confuse, est indispensable soit pour donner des éléments d'explication des phénomènes étudiés soit pour les situer. Le contexte apparaît alors comme une construction complexe émanant de chercheurs qui travaillent à comprendre des phénomènes, chacun en proposant sa propre définition en fonction des objets qu'il entend observer. Toutefois, si cette notion est sujette à variations, des tendances se dégagent qui permettent de proposer des pistes pour caractériser plus finement le contexte dans le cadre des recherches sur les activités informationnelles.

Mots-clés : contexte, activités informationnelles, usages, pratiques informationnelles, activité professionnelle.
This article proposes to study the notion of context in research on information activities. A review of the literature shows that this notion, while often vague and confused, is essential as an explanatory element or as a means of situating the phenomena under consideration. The notion of context constitutes a complex construction. Each researcher proposes his or her own definition according to the phenonema he or she seeks to observe and to understand. However, while this notion is subject to variations, certain trends can be identified which allow us to characterize more precisely the notion of context within the framework of research on information activities.

Keywords: context, information practices, information uses, professional activities. 
La réflexion proposée dans cet article s'appuie sur un triple constat. Le premier est relatif aux activités informationnelles. Ces activités, effectuées en lien avec des dispositifs info-communicationnels, des documents, ou des ressources informationnelles, et qui consistent à chercher, produire, analyser des informations, sont devenues, lorsqu'elles sont menées par des acteurs non spécialistes (nous entendons ici «non professionnels du secteur de l'information-documentation ») chronophages (Guyot, 2013) et prennent de plus en plus de place et de temps dans leur quotidien. Ainsi, Isaac et al. (2007) montrent que les technologies de l'information et de la communication recomposent, au sein des organisations, l'espace-temps des acteurs et entrainent une surcharge informationnelle les amenant à consacrer davantage de temps à la recherche d'informations tout en réduisant, par conséquent, le temps nécessaire pour leur traitement.

Le deuxième constat qui s'impose est le suivant: les dispositifs qui permettent ces activités sont de plus en plus nombreux et variés. Ils peuvent être élaborés en interne ou être externes aux organisations; ils peuvent être conçus spécifiquement pour accompagner les activités des acteurs concernés ou ne pas être dédiés à un type d'application précise. Staii en constatant que "l'information est de plus en plus présente en contexte professionnel» (Staii, 2013, 43) fait état d'une triple tendance qui caractériserait l'informationnalisation' dans les organisations: "une diffusion massive et une diversification des TIC en usage, une inscription de plus en plus profonde des TIC dans les activités sociales et professionnelles, une croissance des flux et une diversification des activités informationnelles» (Staii, 2013, 33). Si les dispositifs destinés à des activités info-communicationnelles sont de plus en plus nombreux dans les organisations, ils ne sont pas toujours pertinents et leur usage est bien souvent partiel (Comtet, 2007).

Enfin, le dernier constat est lié aux conséquences de la multiplication et de la complexification des dispositifs et ressources informationnelles: pour intégrer ces outils dans leurs pratiques, les acteurs sont amenés à développer des compétences et savoir-faire de différentes natures. D'une part des compétences techniques relatives à l'utilisation des dispositifs disponibles et à la capacité de s'adapter à de nouveaux outils de manière fréquente et rapide. Et d'autre part des compétences info-documentaires liées à la recherche d'information, la gestion de documents et d'informations sur différents supports, la capacité d'analyse, d'évaluation, la mise en place de systèmes d'information en vue du stockage, de la réutilisation ou encore du partage avec d'autres membres de la communauté professionnelle, que ce soient les services ou l'organisation dans son ensemble (Guyot, 2002) (Paganelli, 2013). Plus généralement, comme

1 Nous entendons ici l'informationnalisation au sens que Bernard Miège lui attribue en 2007: comme "une logique sociale qui se caractérise par la circulation croissante et accélérée des flux d'information éditée ou non dans la sphère privée, dans celle du travail et dans l'espace public» (Miège, 2007, 66). 
I'indique Bernard Miège, les pratiques des acteurs sont sous-tendues par l'émergence de nouvelles normes d'action communicationnelles en relation avec les TIC: le déplacement des sphères d'action et notamment l'interpénétration entre sphère privée et professionnelle; l'articulation entre outils, réseaux et contenus; l'individualisation des pratiques info-communicationnelles, qui requièrent de nouvelles compétences dans l'organisation de l'activité de travail comme dans les collectifs de travail. Ces nouvelles normes sont à la fois prescrites par les organisations et les concepteurs des dispositifs (Miège, 2007).

Parce que ces activités se transforment et se complexifient, les recherches qui leur sont consacrées, qu'elles les abordent sous l'angle du processus de recherche d'information, des modalités d'accès à l'information ou encore des usages ou pratiques informationnelles, sont nombreuses. Beaucoup s'inscrivent en sciences de l'information et de la communication mais d'autres disciplines s'emparent également de la question: c'est le cas de l'informatique, des sciences de la gestion, des sciences du langage ou de la psychologie. Leurs auteurs poursuivent des objectifs divers: certains visent une modélisation et la conception ou l'amélioration de dispositifs techniques alors que d'autres cherchent à comprendre et expliquer des phénomènes observés.

Quelle que soit l'approche privilégiée, la notion de contexte y est très souvent mobilisée. Elle l'est pour plusieurs raisons: pour préciser le cadre dans lequel les activités étudiées s'effectuent; pour évoquer les facteurs qui l'influencent; plus largement pour se dégager d'une approche techno-centrée qui a fait la part belle, durant de nombreuses années, aux aspects techniques liés aux activités informationnelles.

Cet article propose ainsi de faire le point sur la notion de contexte. Des revues de la littérature ont déjà été proposées sur cette question en sciences humaines et sociales, et notamment en psychologie (Grossen, 2001), en sociologie (Raynaud, 2006) ou en socio-linguistique (Schmoll, 1996). Ici, nous nous attacherons plus spécifiquement à la convocation de cette notion dans les études inscrites en sciences de l'information et relatives aux activités informationnelles dans le cadre professionnel.

Nous dresserons, dans un premier temps, une revue de la littérature, montrant que cette notion, en partie floue et souvent confuse, apparaît toutefois indispensable; ce panorama nous amènera à considérer le contexte comme une construction émanant du chercheur. Nous proposerons enfin des pistes conceptuelles et méthodologiques permettant de cerner la notion de contexte telle qu'elle nous semble utile et fructueuse pour une étude des activités informationnelles au travail. 


\section{1. \\ Le contexte: une notion largement mobilisée dans les études relatives aux usages et pratiques informationnelles}

Nombreux sont les travaux qui évoquent aujourd'hui la recherche d'information contextualisée, contextuelle ou située ou encore l'analyse de l'accès à l'information ou des pratiques informationnelles en contexte comme l'ont montré notamment Courtright, 2007, Chaudiron et al., 2010, Boubée et al., 2010, Grivel, 2011, Munisk, 2012, Case, 2012. Le contexte y est, le plus souvent, caractérisé: contexte scolaire ou universitaire (Aillerie, 2011, Boubée et al., 2011, Cordier, 2011), professionnel (Taylor, 1991, Thivant, 2005, Paganelli et al., 2009, Huvila, 2008b) et organisationnel (Guyot, 2002 et 2013, Ihadjadene et al., 2010), ou encore le contexte de la vie quotidienne (every day life) (Savolainen, 2004). L'utilisation du terme contexte permet également de préciser de manière encore plus fine un domaine d'application dédié: I'on parlera ainsi de recherche d'information en santé, dans le tourisme, dans le cadre des loisirs, etc.

Pourtant, la prise en compte de la notion de contexte dans les études du domaine semble relativement tardive (Boubée et al., 2010, 66); les premiers colloques et publications intervenant pour l'essentiel, dans le champ des sciences de l'information, dans les années 90 par Belkin (1995), Ingwersen (1996), Leckie et al. (1996) ou Saracevic (1997) notamment.

\subsection{Des objectifs multiples}

La notion de contexte est mobilisée avec des objectifs multiples. Elle peut l'être dans une perspective modélisante. Par exemple, en informatique, les travaux qui s'intéressent la recherche d'information contextuelle sont légion. Ils visent alors la conception ou l'amélioration de systèmes en prenant en compte la diversité des contextes (et notamment des utilisateurs et de leurs «besoins d'information "). C'est le cas notamment, mais non exclusivement, des travaux menés au sein de I'IRIT ${ }^{2}$. Quand l'objectif des recherches est de développer ou d'améliorer des systèmes de recherche d'information, le contexte est défini de manière à ce que les différents éléments qu'il recouvre puissent être modélisés et intégrés dans le système. Ainsi, «le contexte fait référence aux connaissances relatives, aux intentions de l'utilisateur (tâche à accomplir, perception de la tâche, type d'information recherchée), à l'utilisateur lui-même (connaissance a priori, profil, culture), à son environnement (environnement matériel, historique des tâches), au domaine du besoin en information (nature du corpus, domaines abordés) et aux caractéristiques du système (représentation des documents, méthode d'appariement requête/document, interface de visualisation, stratégies 
d'accès à l'information)»(Hernandez, 2005).

En sciences de l'information ${ }^{3}$, les recherches s'appuyant sur une analyse du contexte relèvent d'une approche épistémologique orientée usagers. Se distinguant d'une approche orientée système qui prévalait dans les études portant sur ces questions jusque dans les années 90, le «paradigme usager considère donc que l'attention doit être davantage portée sur les besoins réels de l'usager et son environnement " (Chaudiron et al., 2010, 4), les recherches qui en relèvent envisagent les pratiques informationnelles comme des processus prenant place dans un contexte spécifique (Courtright, 2007). Les auteurs, notamment anglo-saxons, dont les travaux s'inscrivent dans cette approche proposent une modélisation cognitive du processus de recherche d'information, des comportements informationnels ou plus largement de l'activité informationnelle. De nombreux modèles ont ainsi été développés pour analyser et décrire le processus de recherche d'information et d'accès à l'information (Ellis, 1989; Krikelas, 1983; Kulthau, 1991 et Wilson, 1981 notamment). Ces modèles cherchent à expliciter un processus, ses causes et ses conséquences, ils visent ainsi à donner une "vision simplifiée de la réalité» (Case, 2012) et fournissent un cadre pour penser et analyser un problème (Wilson, 1999). De manière générale, ces modèles envisagent l'activité d'information comme un processus composé d'un certain nombre d'étapes et soumis à diverses influences. Sur ce sujet, un état de l'art récent et très complet est proposé par Donald Case (2012). Parmi ces modèles, certains insistent sur la dimension contextuelle du processus de recherche d'information. C'est le cas du travail de Leckie et al. (1996) qui proposent un modèle du processus de recherche d'information dans lequel les rôles professionnels influencent l'activité informationnelle ou encore de Cheuk (1999). D'autres auteurs se sont attachés à modéliser la recherche d'information pour des professions particulières: Ellis (1989 et 1993) s'est intéressé aux scientifiques, Fidel et al. (2004) aux ingénieurs, Choo (2007) aux managers, par exemple. Le contexte est alors mobilisé, la plupart du temps, pour comprendre et pour déterminer les facteurs qui influent sur l'activité informationnelle. Parler de contexte revient alors à considérer que les acteurs peuvent se trouver dans une diversité de situations; que ces situations influencent les pratiques informationnelles et que l'analyse et la prise en compte des caractéristiques du contexte permettent une meilleure connaissance et compréhension des pratiques informationnelles.

La notion de contexte permet alors de préciser le cadre dans lequel les phénomènes étudiés prennent place et ainsi de situer les matériaux recueillis, ou encore de proposer des variables explicatives aux résultats observés. 
Ingwersen et Järvelin dans un ouvrage consacré à la recherche d'information en contexte (2005) ${ }^{4}$, ou Johnson dans un article paru en 2003 et intitulé On contexts of information seeking, soulignent l'importance de la prise en compte du contexte. Elle l'est d'autant plus que la plupart des recherches sur ces questions s'appuient sur des études de terrain qualitatives pour lesquelles la contextualisation permet de guider l'interprétation des données, et ainsi de situer les matériaux sur lesquels elle s'appuie et, du même coup, les propos et les résultats des chercheurs. La contextualisation est entendue comme une mise en relation entre des observations et le cadre dans lequel elles ont été effectuées. Ainsi, comme le suggère Goffman dans Les cadres de l'expérience en 1974, le contexte est construit pour rendre les faits sociaux intelligibles, les observer, les décrire, et enfin les interpréter. En sciences sociales, le contexte joue un rôle dans de nombreuses explications sociologiques (Raynaud, 2006).

On retrouve la même préoccupation chez les chercheurs en sciences de l'information et de la communication. Ainsi, pour définir l'approche communicationnelle, qui permet d'étudier ce qu'il nomme les «faits de communication", Mucchielli (2006) évoque comme incontournable la notion de contextualisation. En s'appuyant notamment sur les travaux de Bernard Miège (2004) et de Dominique Carré (2002), il développe la notion de situation qu'il considère comme englobante par rapport au contexte. Ainsi les phénomènes de communication interviendraient dans une situation qui serait marquée par quatre contextes principaux: contexte des normes, des enjeux, des positionnements et des relations, ainsi que par des éléments spatiaux, temporels, sensoriel-physiques qui participeraient de cette contextualisation. Les pratiques info-communicationnelles seraient alors nécessairement inscrites dans une situation, dans un contexte donné.

Parler de contexte permet donc de décrire le cadre dans lequel se passent les activités et phénomènes étudiés. Dès1981, Tom Wilson assimile le contexte à un cadre permettant de comprendre et d'interpréter l'activité de recherche d'information. Toutefois, dans cet article, l'auteur ne définit pas précisément le contexte mais l'assimile à ce qu'il appelle «l'univers de connaissance" (knowledge universe) constitué des ressources informationnelles, du système d'information et du «monde de l'usager». Donald Case (2012) définit le contexte comme la combinaison de facteurs personnels et situationnels, qui sert de cadre à l'activité d'information. Pour Huvila (2008a) qui développe une approche orientée tâche, dans le milieu professionnel, le contexte fait référence à l'environnement auquel la tâche appartient. L'environnement est alors défini généralement comme un environnement concret (faisant référence aux ressources informationnelles disponibles, aux individus...) et abstrait (se

4 Ingwersen Peter and Kalervo Järvelin (2005). The Turn: Integration of Information Seeking and Retrieval in Context, Dordrecht: Springer: Springer e-books, 2005. 
référant aux normes et valeurs de l'organisation, aux contraintes, aux buts...). Byström et al. (2005) ont proposé de décrire le cadre dans lequel la tâche de recherche d'information peut s'effectuer, selon trois niveaux: (1) le contexte de travail de la tâche qui est représenté par les ressources, les contraintes, la culture de l'organisation; (2) le contexte de la recherche d'information en lien avec le besoin initial d'information; (3) le contexte de la recherche d'information qui correspond à l'activité de recherche proprement dite ainsi qu'au traitement des résultats de recherche. Les contextes apparaissent ici comme des cadres dans lesquels les actions sont menées, ces cadres étant définis par rapport à la fonction qu'il occupe.

Définir le contexte permet également de mettre en évidence les éléments qui le composent comme des variables explicatives des phénomènes étudiés. Bryce Allen (1997), dont les travaux portent sur la recherche d'information, propose d'identifier les variables qui constituent le contexte et distingue des variables individuelles et sociales qui interagissent entre elles et qui permettent de comprendre et d'interpréter les besoins d'information des usagers. Ces propositions ont ensuite été reprises par différents chercheurs dont notamment (Pettigrew et al., 2001; Cool, 2001) pour définir un contexte pour la recherche d'information et trouver, à partir de là, des éléments d'explication aux comportements et pratiques de recherches d'information ou aux besoins informationnels.

Parler de contexte permet, notamment dans les recherches relatives spécifiquement à la recherche d'information, de considérer que ce processus est influencé par un certain nombre de facteurs. Le contexte, et les éléments qui le composent, sont entendus comme ayant une incidence sur le phénomène étudié. En sociologie, Bernard Lahire (2001) suggère ainsi que les pratiques individuelles sont le résultat de dispositions sociales (expériences, vécu) et de contraintes contextuelles qui pèsent sur les acteurs. Considérer que le contexte influence et contraint les activités informationnelles, nécessite alors de préciser ce qui relève du contexte. Dans les recherches qui ne visent pas une modélisation, le contexte est souvent défini de manière assez large. Ainsi, Tom Wilson dans le model of information behavior qu'il proposait en 1981 (Wilson, 1981) définissait la notion de contexte "context of information seeking" comme un ensemble complexe en lien avec le monde de l'usager (comprenant notamment son monde professionnel), le système d'information et les ressources informationnelles. Ces différents éléments, en interaction les uns avec les autres, influençant les besoins d'informations et les comportements des usagers. Quelques années plus tard, Robert Taylor définit, d'une manière qui nous semble un peu plus précise, le contexte qu'il appelle «information use environment » et qu'il envisage comme un ensemble d'éléments qui influencent la circulation de l'information entre les individus ou les groupes d'individus, l'usage qui en est fait, et qui détermine les critères par lesquels la valeur de l'information sera jugée. Il complète cette présentation en précisant qu'il s'agit d'un contexte ou une situation dans laquelle les individus vivent et travaillent, et où ils font des choix concernant 
les informations qui leur sont utiles ${ }^{5}$ (Taylor, 1991). Ce contexte (information use environment) s'organise autour de trois entités: I'environnement géographique, l'environnement organisationnel et enfin l'environnement social et culturel. Ayant étudié les activités informationnelles en contexte professionnel, Taylor a particulièrement développé l'environnement organisationnel, considérant qu'il se compose de quatre entités: (1) des groupes d'individus (caractérisés par leurs professions, groupes sociaux, expertise, pratiques sociales...), (2) des situations problématiques propres à chaque groupe d'usagers d'information et qui influencent la recherche d'information, (3) le contexte structurel qui entoure ces groupes d'usagers (situations de travail, culture informationnelle de l'organisation...) et enfin (4) les stratégies de résolution de situations problématiques et les modalités de prise de décision. Howard Rosenbaum (1996), qui a repris et approfondi les travaux de Robert Taylor, propose, lui, de considérer le contexte, dans le cadre des organisations, comme composé d'éléments matériels, techniques et sociaux. Parmi ces éléments, un ensemble de règles procédurales ou techniques, liées aux activités menées au sein de l'organisation, ainsi que des ressources comme le sont les dispositifs ou documents. Ces éléments, selon l'auteur, affectent les pratiques informationnelles. Enfin Mizzaro (1997), dans l'article «Relevance: the whole history» propose trois dimensions pour caractériser la pertinence: le sujet (sur lequel porte la recherche), la tâche (que l'usager va réaliser avec les documents et informations trouvés) et le contexte. Il le définit de la manière suivante: relève du contexte tout ce qui ne concerne ni le sujet de la recherche ni la tâche mais qui affecte la manière dont la recherche est menée et l'évaluation des résultats. II cite notamment les documents déjà connus, le temps et le budget disponibles.

Ce rapide état de l'art met en évidence deux approches du contexte: l'une sociale et l'autre orientée autour de l'activité cognitive des individus. L'approche sociale envisage le contexte sous l'angle d'aspects temporels, spatiaux ou liés à l'organisation dans laquelle les activités informationnelles se situent. L'approche orientée activité, elle, porte l'attention sur les aspects cognitifs liés aux caractéristiques de l'individu et de la tâche qu'il a à mener. Dans ce cas, la référence à la théorie de l'action située développée par Lucy Suchman (1987) est parfois explicite (Anderson, 2007, Tamura et al., 2007, Xie, 2007). Il apparaît également que les éléments constitutifs du contexte sont envisagés comme juxtaposés sans que soit toujours proposée une vision globale des interactions entre eux. Ces éléments sont, le plus souvent, assimilés à des contraintes qui affectent les activités informationnelles.

\subsection{Une notion indispensable mais souvent floue}

Si le recours à la notion de contexte semble incontournable pour la plupart

5 "Context or setting within which people live and work and where they 'make choices about what information is useful to them at particular times'», p. 218. 
des auteurs de recherches consacrées aux usages et aux pratiques informationnelles, elle n'en demeure pas moins délicate à cerner car souvent présentée de manière vague et imprécise. Ce constat confirme ce que suggère Bernard Lahire (1996) pour qui la notion de contexte est, en sciences sociales, à la fois l'une des plus floues et l'une des plus utiles.

La notion est le plus souvent implicite dans les travaux de recherche, le contexte étant considéré sans être défini. Brigitte Simonnot parle ainsi de «fourre-tout» lorsqu'elle suggère que le contexte est une notion qui «demanderait à être définie de manière plus fine» (Simonnot, 2002). Byström et Hansen procèdent à la même analyse lorsqu'ils évoquent le contexte comme «[...] vaguely understood and used in various ways in information studies " (Byström et al., 2005). Christina Courtright (2007) fait le même constat lorsqu'elle analyse le concept de contexte dans la littérature anglo-saxonne sur les usages de I'information: "[...] context as a concept appears in the research literature as largely amorphous and elusive». Enfin Chaker et al. (2010) considèrent qu'il n'existe pas de définition unique du contexte ou de ses composantes dans le domaine de la recherche d'information.

Par ailleurs, il ne semble pas y avoir de consensus sur ce qui est entendu par contexte ou, en tous cas, pas de définition clairement posée et partagée. Brenda Dervin (1997) citée par Donald Case (2012) pointe les limites de cette notion dans les études relevant des sciences de l'information. Elle montre ainsi que, si le terme est largement utilisé, il est très peu défini ou il l'est de manière extrêmement variable, pouvant être utilisé en référence aux notions de culture, d'organisation, de comportement, ou encore de situation.

Ainsi, d'autres termes sont parfois utilisés: milieu, environnement, cadre et, le plus souvent dans les études anglo-saxonnes situation comme l'écrit Coleen Cool dans un article consacré à ce sujet (Cool, 2001). L'auteur met en évidence l'importance de ces deux notions (contexte et situation) dans les études relatives à la recherche d'information, aux comportements de recherche des usagers ou encore à l'interaction entre les usagers et les systèmes. L'auteur montre également que ces termes sont utilisés aussi bien dans des perspectives cognitive, interactionniste et sociale. Cet article confirme, comme pour la notion de contexte, la multiplicité des définitions du concept de situation. II semble, toutefois, que la notion de situation soit davantage liée aux acteurs et à leurs buts, à leurs activités, ou à leurs habilités, quand le terme contexte est, en général, entendu comme faisant référence à un environnement plus large, constitué de facteurs et de variables qui affectent le processus d'information.

Si la notion de contexte semble floue, c'est, le plus souvent, parce qu'elle n'est pas définie par les auteurs qui l'utilisent ni dans ses contours ni dans ce qu'elle recouvre. Ainsi, le contexte peut faire référence au champ social, au champ institutionnel, à la sphère privée ou publique. II peut être ainsi appréhendé à différents niveaux, micro, méso ou macro, selon les échelles d'observation privilégiées. Le contexte peut faire référence, selon les cas au pays (le contexte 
français, le contexte nord-américain...), à une communauté professionnelle (le contexte de l'entreprise, le contexte bancaire, universitaire, scolaire...), au service d'une organisation (les ressources humaines, la communication...), dont les contours ne sont pas toujours dessinés clairement. Lorsque l'on évoque le contexte scolaire par exemple, comment est-il envisagé: en tant que lieu physique (établissement scolaire)? En tant que porteur de situation (situation d'apprentissage)? Ou comme lieu de relations et de pouvoirs (entre élèves et enseignants par exemple)? Et quels sont les éléments considérés comme spécifiques de ce contexte que le chercheur mettra en relation avec les résultats observés?

Enfin, il arrive que le terme en contexte soit utilisé pour indiquer que les phénomènes étudiés sont situés sans plus de précision mais peut-être simplement pour signaler que le chercheur envisage l'analyse de ces observations en relation avec le cadre dans lequel elles s'inscrivent.

\section{2.}

\section{Le contexte: une construction complexe}

En nous appuyons sur les travaux cités précédemment comme sur nos propres recherches, et sans ambition d'avoir réalisé un tour complet de la question, nous proposons de considérer le contexte comme une construction du chercheur, construction qui permet d'ancrer les recherches et études de terrain dans une réalité, d'analyser et d'interpréter les résultats, enfin de mettre en relation les pratiques et activités observées avec l'ensemble des circonstances qui les accompagnent. Ainsi, "le contexte n'existe pas en soi, il est le prisme à partir duquel nous pouvons interpréter et agir sur notre environnement» (Broudoux, 2012).

Ici, notre propos est de tenter de cerner la notion de contexte telle qu'elle nous semble utile et fructueuse pour une étude des activités informationnelles au travail.

\subsection{Une approche par l'activité}

Définir le contexte permet de circonscrire le champ d'étude. La plupart des travaux évoquant la notion de contexte situent leurs observations dans un cadre particulier et souvent professionnel. Selon Christina Courtright (2007), il est en effet plus facile de délimiter les contours du contexte dans ce cadre que dans celui, beaucoup plus général, du quotidien et de «la vie de tous les jours». Nous inscrivons ici plus précisément notre réflexion dans une approche orientée activité, considérant que la question du contexte prend tout son sens dans les études relevant d'une telle approche.

Nous considérons en effet les activités comme situées et émergeant du contexte (ou des circonstances) dans lequel elles prennent place, faisant 
référence ainsi à Lucy Suchman lorsqu'elle écrit: "J'introduis l'expression 'action située' pour souligner que tout cours d'action dépend de façon essentielle de ses circonstances matérielles et sociales" (Suchman, 1987, 50, traduit par Béguin et al., 2004). Pour Pascal Beguin et Yves Clot, écrire que l'action émerge des circonstances signifie deux choses pour Suchman: l'action dépend des circonstances (indexicalité), et le fait d'agir définit le contexte de l'action (réflexivité).

Parler de l'activité consiste, de notre point de vue, à parler des acteurs au travail et à les considérer comme des individus ayant des pratiques qui leur sont propres mais également comme des individus engagés dans un collectif organisé avec des règles plus ou moins explicites, des manières de faire individuelles et partagées. "L'activité qu'est le travail est collective, organisée; et [...] comme toute activité collective, elle est faite de sens et de non sens, de projets et de routines, de partage et de rien qu'à moi »(Delcambre, 2007).

C'est donc dans cette approche que nous situons notre réflexion sur une définition du contexte qui pourrait être partagée par les chercheurs de manière à comparer des situations, à mettre en regard des résultats et à, ainsi, proposer des connaissances communes sur la question.

\subsection{Un ensemble d'éléments en interaction}

Nous considérons alors que le contexte repose sur un ensemble d'éléments interagissant entre eux. Ces éléments, que nous détaillons plus loin, concernent les acteurs, les activités multiples dans lesquelles ils sont impliqués, l'environnement informationnel et enfin l'environnement organisationnel dans lequel elles prennent place.

\section{Les acteurs}

Les acteurs qui agissent au sein des organisations participent du contexte qui structure leurs activités informationnelles. Ce sont eux qui travaillent avec I'information et un certain nombre de variables les concernant influencent leurs «manières de faire». Ils se caractérisent d'abord par le fait qu'ils agissent dans une situation particulière: une situation de travail. Ce ne sont pas les individus en tant que tel qui constituent le contexte mais les individus en situation d'agir. Lorsqu'ils agissent, les acteurs tiennent un rôle: dans La présentation de soi (1956), Erving Goffman assimile le monde à la scène d'un théâtre où les individus sont des acteurs qui tiennent des rôles, et les relations sociales des représentations soumises à des règles déterminées et précises. Ici, les stratégies développées par les acteurs sont liées au fonctionnement de l'organisation, à sa structure hiérarchique, aux relations qui y sont nouées. Enfin, le rôle des acteurs n'est pas figé, il évolue au fur et à mesure que ces derniers agissent. II évolue dans un temps court (l'activité au quotidien) mais aussi sur une période plus longue (le déroulement de carrière par exemple). Ainsi, dans le cadre des activités informationnelles, le rôle de l'acteur évoluera de chercheur d'informations à celui de lecteur, d'évaluateur, de producteur... Le terme d'acteur 
est ici choisi en référence notamment aux écrits de Brigitte Guyot (2004a; 2004b) car il met l'accent sur l'individu actif, doté d'une intentionnalité et d'une responsabilité lui permettant de faire des choix et d'endosser différents rôles (producteur, lecteur...).

Ce qui définit les acteurs, c'est donc le fait qu'ils sont en train d'agir mais ce sont également un certain nombre de variables liées à leur situation au sein de l'organisation: des facteurs cognitifs relatifs au contexte de travail (expertise, savoir-faire, univers de connaissances, rôle professionnel, statut), des variables démographiques, les usages qu'ils font des ressources informationnelles disponibles et plus largement leurs pratiques info-communicationnelles (notamment en matière de recherche d'information, de lecture...).

\section{Les activités dans lesquels ils sont engagés}

Le contexte se construit par rapport aux activités des acteurs, et notamment celle que nous considérons ici comme activité principale et qui les amène à rechercher, produire, analyser des informations. Cette préoccupation fait écho à différentes études en sciences de l'information consacrées à ce que les auteurs dénomment la tâche. Des recherches récentes, dans la littérature anglo-saxonne, se revendiquent comme des études orientées tâche (task oriented) (Byström, 2007) et analysent I'influence de la tâche et du but principal des acteurs sur leur activité de recherche d'information et plus spécifiquement sur leurs attentes, leur comportement, l'utilisation qu'ils font des sources d'information mais également les représentations qu'ils en ont. Dans ces études, la tâche est considérée comme un ensemble d'activités dynamique, orienté par un but et clairement identifié comme un processus borné par un commencement et une fin. Elle est soumise à certaines contraintes et exigences, elle peut être exécutée par un individu de manière isolée ou nécessiter la coordination de plusieurs acteurs.

Dans cette acception, l'activité se distingue d'abord par un ou plusieurs objectifs à atteindre et par des contraintes qui lui sont spécifiques: le délai, les résultats attendus, les contraintes matérielles (en termes financier, d'outils et de dispositifs nécessaires...), ou les conditions de réalisation, selon que l'activité est menée en contact avec des personnes externes (clients, patients, fournisseurs...) ou uniquement au sein de l'organisation. Elle s'étudie également par le lien qu'elle entretient avec l'information et la nécessité plus ou moins forte qu'elle implique de s'informer ou de produire des informations.

L'activité se caractérise aussi par son déroulement, les différentes tâches qui la composent et la manière dont elles s'agencent, la temporalité dans laquelle elle se déroule, la répétitivité éventuelle. Pour autant, il n'est pas toujours évident de distinguer clairement les tâches, d'en préciser l'articulation, d'en dégager des bornes temporelles.

Enfin, l'activité principale s'étudie par rapport au reste de l'organisation, à 
la manière dont elle s'articule avec les activités des autres acteurs, ou encore à la place qu'elle occupe dans l'ensemble et à l'importance qu'elle y tient, ou les enjeux qu'elle y représente.

Mais considérer l'activité principale des acteurs c'est aussi la relier à l'ensemble des activités menées au sein de l'organisation et l'envisager dans sa relation à celle de l'organisation dans son ensemble.

\section{L'environnement informationnel}

Le contexte est également construit par l'environnement informationnel qui entoure les activités informationnelles développées au sein de l'organisation.

L'environnement informationnel est constitué des documents et dispositifs qui sont disponibles, au sein de l'organisation comme à l'extérieur, pour permettre aux acteurs de développer leurs activités informationnelles. De nombreux documents produits en interne ou achetés à l'externe sont accessibles, ainsi que différents dispositifs qui permettent aux acteurs de chercher des informations ou d'en produire. Certains de ces outils sont développés en interne et sont spécifiques à l'organisation, alors que d'autres sont accessibles publiquement.

Enfin, l'environnement informationnel est structuré par la manière dont I'information est gérée dans l'organisation: présence ou non d'un service de documentation et de professionnels du secteur, existence ou non de projets dédiés à la gestion et au traitement des informations que ce soit sous la forme de projets de numérisation, de gestion des connaissances, ou relatifs au records management.

\section{L'environnement socio-organisationnel}

Les activités informationnelles prennent place au sein d'une communauté, l'organisation, régie par un certain nombre de règles, de normes ou encore de valeurs qui les influencent. Plusieurs éléments constituent l'environnement socio-organisationnel dans lequel l'acteur évolue: la manière dont l'entreprise s'organise (hiérarchie, services, fonctions), les méthodes de management, les stratégies et politiques développées, les relations entre les différents acteurs qui y travaillent. Sont également constitutifs de l'environnement organisationnel, la culture d'entreprise (que l'on définit ici rapidement comme l'histoire et les valeurs partagées), ou la culture informationnelle qui est développée au sein de l'organisation.

2.3. Un cadre d'analyse pour étudier les activités informationnelles «en contexte»

Les éléments précédemment cités peuvent s'envisager à trois niveaux: le niveau micro (relatif à l'individu et à son quotidien), méso (en lien avec l'organisation) et macro (à l'échelle de la société et des mutations qui l'affectent et influencent ainsi les pratiques sociales). 
Par ailleurs, parmi ces éléments, certains apparaissent stables alors qu'autres seraient dynamiques et évolutifs. L'inscription spatio-temporelle représenterait ainsi un ancrage stable: tout phénomène prenant place dans un espace et dans une période agissant sur les évènements considérés. Mais le contexte ne serait pas totalement immuable ni déterminé a priori et évoluerait en fonction des actions menées, des stratégies et tactiques développées, des relations de pouvoir dans lesquelles sont impliqués les acteurs concernés. Les acteurs joueraient ainsi sur leur environnement en le faisant évoluer.

Enfin, le contexte se caractériserait d'une part par des éléments relevant d'une temporalité immédiate et d'autre part par des éléments structurants inscrits dans une temporalité plus longue. Apparaissent ainsi comme constitutifs du contexte, ces logiques sociales que Bernard Miège (2004) définit comme "des mouvements structurants-structurés de longue durée», "des logiques transversales fortes et structurales"; ce sont des tendances ou changements qui caractérisent l'ensemble du champ de l'information et de la communication et qui expliquent et déterminent pourquoi les acteurs agissent et interviennent dans tel ou tel sens. Le contexte dans lequel les activités informationnelles prennent place est ainsi marqué par ces changements qui touchent notamment à l'édition et la production d'informations spécialisées, à la place des informations dans l'ensemble des champs sociaux, aux pratiques sociales.

En termes de méthodologie, dans le cas d'études de terrain visant à étudier les activités informationnelles en contexte, les entrées à analyser pourraient donc être regroupées de la manière suivante:

$\times$ Les acteurs du point de leurs caractéristiques individuelles (expertise, poste occupé...), de leurs pratiques info-communicationnelles, que ce soit dans la sphère privée ou professionnelle mais aussi dans leur dimension collective (relations qui se nouent entre acteurs, interaction entre action individuelle et collective);

× L'activité principale de l'acteur, analysée dans sa temporalité, dans sa relation avec les autres activités menées au sein de l'organisation, comme moteur des activités informationnelles mises en œuvre, et la manière dont ces activités multiples se confondent dans le quotidien des acteurs;

$\times$ Les dispositifs et documents dans leurs dimensions sociales, symboliques, techniques, dans leurs modalités d'accès à l'information;

× Enfin, les enjeux à l'œuvre dans les entreprises du point de vue de leur organisation, des relations entre acteurs, des normes et valeurs affirmées, et de la culture informationnelle qui y est développée.

Chacune de ces entrées serait analysée avec un triple regard portant une attention particulière:

× aux niveaux micro, méso et macro. Ainsi, par exemple l'acteur serait envisagé dans sa dimension individuelle, collective (au sein du service dans lequel il travaille, de l'organisation) et sociale. Les dispositifs 
info-communicationnels et les documents étudiés seraient de trois types: ceux crées au sein de l'organisation pour accompagner l'activité des acteurs; les dispositifs externes utilisés par les acteurs de l'entreprise; les dispositifs personnels et mis en place par chaque acteur pour ses propres besoins.

× à la temporalité, de manière à analyser les observations immédiates en ayant à l'esprit les logiques structurantes qui modèlent et affectent les pratiques info-communicationnelles.

× à la stabilité en distinguant des constituants stables et ceux qui évoluent et peuvent se voir modifiés par l'action.

Mobiliser la notion de contexte, lorsque l'on étudie les activités informationnelles, suppose, pour le chercheur ou le praticien, qu'elle soit définie et précisée notamment dans ses contours et dans ce qu'elle recouvre. La plupart du temps, les recherches concernées s'appuient sur des études de terrain. Dans ce cadre, il apparaît donc nécessaire que soit proposée une grille d'analyse méthodologique permettant de prendre en compte le contexte tout en étant précis sur ce qui en relève.

\section{3. Conclusion}

La revue de littérature exposée précédemment met en évidence plusieurs éléments:

× la fréquente utilisation de la notion de contexte dans les études portant sur les activités informationnelles, qu'elles soient envisagées sous l'angle du processus de recherche d'information ou des usages et pratiques informationnelles. Recourir au contexte permet de faire émerger des facteurs explicatifs des phénomènes étudiés ou de situer le cadre dans lequel ils interviennent;

× cette notion apparaît indispensable mais est le plus souvent floue et peu explicitée. Indispensable car étudier les activités informationnelles en s'appuyant notamment sur des études de terrain implique, incontestablement, de situer ces activités. Peu explicitée, sûrement car elle apparaît la plupart du temps, pour les chercheurs qui la mobilisent, comme une donnée acquise et évidente, qu'il n'est pas nécessaire de définir.

Nous suggérons ici que le contexte est une construction émanant de chercheurs qui travaillent à comprendre et à expliquer des phénomènes, chacun en proposant sa propre définition en fonction des objets qu'il entend observer et des mises en relation auxquelles il envisage de procéder. Toutefois, si cette notion est peu définie et sujette à variations, il semble utile de définir le contexte, d'en délimiter les frontières, et d'en considérer les éléments qui le constituent. Les études portant sur les usages et pratiques en sciences de l'information, 
si elles sont nombreuses, riches et variées, permettent difficilement de faire émerger des connaissances générales tant les méthodologies mises en œuvre, les objets ou populations étudiées, varient (Paganelli, 2012). Le même constat s'impose lorsque l'on s'intéresse aux études évoquant la notion de contexte. En donner une définition précise nous semble être un préalable indispensable pour parvenir à un cadre, une grille d'analyse qui permettrait de comparer des observations, de transposer des résultats et, finalement, de voir l'émergence des connaissances générales et partagées sur la question. 
Bibliographie

Aillerie K. (2012). Pratiques informationnelles informelles des adolescents (14-18ans) sur le web. Thèse de doctorat en Sciences de l'information et de la communication, Université Paris Nord-Paris13, 534 p.

Allen B. (1997). «Information needs: a person-in-situation approach ». In Proceedings of an international conference on Information seeking in context, Tampere, Finland, pp.111-122.

Anderson T.D. (2007). "Settings, arenas and boundary objects: socio-material framings of information practices ". In Information Research, vol.12, n 4 : http://InformationR. net/ir/12-4/colis/colis10.html.

Béguin P. et Clot Y. (2004). "L'action située dans le développement de l'activité». In Activités, vol.1, $\mathrm{n}^{\circ} 2$, pp. 27-49: http://www.activites.org/v1n2/beguin.fr.pdf.

Belkin N. J. (1995). «Anomalous State of Knowledge». In K. E. Fisher, S. Erdelez and E. F. McKechnie(dir.). Theories of information behavior: A researchers' guide. Medford, NJ: Information Today, p. 44-48.

Boubée N. et Tricot A. (2010). Qu'est-ce-que rechercher l'information?, Presses de l'Enssib, 286p. (Papiers. Série Usages des documents).

Boubée N. et Tricot A. (2011). L'activité informationnelle juvénile, Hermès
Lavoisier, 300 p. (Système d'information et organisations documentaires).

BroudouxE. (2012). "Vers l'objet documentaire (re)contextualisé». In $9^{\text {ème }}$ congrès des enseignants documentalistes de l'Éducation Nationale, Objets documentaires numériques: nouvel enseignement?, Paris, Mars.

ByströmK. and Hansen P. (2005). "Conceptual framework for tasks in information studies ». In Journal of the American Society for Information Science Technology, vol.56, n¹0, pp.1050-1061.

Byström K. (2007). «Approaches to 'task' in contemporary information studies ». In Information Research, vol.12, n 4 , October: http://informationr.net/ir/12-4/colis/colis26.html.

CarréD. (2002). La régulation sociale de la diffusion des techniques d'information et de communication. Approche socio-politique et problématique communicationnelle, Mémoire en vue de l'habilitation à diriger des recherches, Université Stendhal, Grenoble3.

Case D. (2012). Looking for information: a survey of research on information seeking, needs, and behavior, Elsevier/Academic Press, 423p.

ChaudironS. et IhadjadeneM. (2010). «De la recherche de l'information aux pratiques informationnelles». In Études de communication, $\mathrm{n}^{\circ} 35$ : http://edc.revues.org/2257.

ChakerH., Chevalier M., Soulé-Dupuy C. et Tricot A. (2010). 
«Gestionnaire de contexte métier: application à la Recherche d'Information ". In VSST'10, Veille Stratégique Scientifique et Technologique, Toulouse, 25-29 octobre.

CheukW.Y.B. (1999). "The derivation of a 'situational' information seeking and use process model in the workplace: employing sense-making ». In International Communication Association annual meeting, San Francisco, California.

Choo C.W. (2007). "Information seeking in organizations: epistemic contexts and contests". In Information Research, vol.12, n²: http://informationr.net/ir/12-2/paper298.html.

Comtetl. (2007). "De l'usage des TIC en entreprise. Analyses croisées entre Sciences de I'information et Sciences de gestion ». In Communication et organisation, $\mathrm{n}^{\circ} 31$, pp.94-107.

CoolC. (2001). "The concept of situation in information science». In Annual review of information science and technology, $\mathrm{n}^{\circ} 35, \mathrm{pp} .5-42$.

Cordier A. (2011). «La recherche d'information sur Internet des collégiens: entre imaginaires, pratiques et prescriptions". In Documentaliste-Sciences de l'information, vol.48, n¹, pp.62-69.

Courtright C. (2007). "Context in information behavior research». In Annual Review of Information Science and Technology, vol. 41, n¹, pp. 273-306.
Delcambre P. (2007). «Pour une théorie de la communication en contexte de travail appuyée sur des théories de l'action et de l'expression». In Communication et organisation, $\mathrm{n}^{\circ} 31$ : http://communicationorganisation.revues.org/108.

Ellis D. (1989). «A behavioural approach to information retrieval design ». In Journal of Documentation, vol.45, pp.171-212.

Ellis D. (1993). "Modeling the information seeking patterns of academic researchers: a grounded theory approach ». In Library Quaterly, vol.6, n³, pp.469-486.

FidelR. and Green M. (2004). "The many faces of accessibility: Engineers' perception of information sources". In Information Processing \& Management, $n^{\circ} 40$, pp.563-581.

GoffmanE. (1956). La Mise en scène de la vie quotidienne, t. $1 \mathrm{La}$ Présentation de soi, Éditions de Minuit, coll. "Le Sens Commun», 1973 pour la traduction française.

Goffman E. (1974). Les cadres de l'expérience, Paris, éditions de Minuit, (coll. «Sens commun»). 1991 pour la traduction française.

Grivel L. (dir.) (2011). La recherche d'information en contexte. Outils et usages applicatifs, Paris, Hermès Science-Lavoisier, coll. Traité des sciences et des techniques de l'information, $288 \mathrm{p}$.

Grossen M. (2001). La notion de contexte: Quelle définition pour 
quelle psychologie? Un essai de mise au point. In Bernié J.P. (dir.) Apprentissage, développement et significations, Bordeaux, Presses Universitaires de Bordeaux, pp.59-76.

Guyot B. (2002). «Mettre en ordre les activités d'information, nouvelle forme de rationalisation organisationnelle». In Les Enjeux de l'information et de la communication, laboratoire Gresec, Université Stendhal, Grenoble: http://w3.u-grenoble3.fr/les_enjeux/2002/Guyot/index.php.

Guyot B. (2004a). «L'activité professionnelle vue par les sciences de l'information". In Revue Hermès, $n^{\circ} 38$, mai, pp.38-47.

GuyotB. (2004b). «Eléments pour une approche informationnelle dans les organisations". In Sciences de la société, $n^{\circ} 63$, octobre2004, 10p.: http://archivesic. ccsd.cnrs.fr/docs/00/06/24/30/ PDF/sic_00001094.pdf.

Guyot B. (2013). «Comprendre la place et les enjeux de l'information dans les organisations". In ClavierV. et PaganelliC. (dir.). L'information professionnelle, Paris, Hermès Science, Lavoisier, pp.199-217.

HernandezN. (2005). Ontologies de domaine pour la modélisation du contexte en recherche d'information, thèse de doctorat en informatique, Université Paul Sabatier, Toulouse, décembre, 248p.: http://www.irit.fr/ Nathalie. Hernandez/nHernandez.pdf.
Huvila I. (2008a). «Information work analysis: an approach to research on information interactions and information behaviour in context ". In Information Research, vol.13, n³, paper 349: http://InformationR. net/ir/13-3/paper349.html.

Huvila I. (2008b). «Work and work roles: a context of tasks». In Journal of Documentation, vol.64, $n^{\circ} 6$, pp.797-815: http:// www.istohuvila.se/files/IstoHuvila_WorkRolesAndTasks_JDOC.pdf.

Ihadjadene M. et ChaudironS. (2010). "Quels modèles pour analyser l'accès à l'information dans les organisations? ». In Les Enjeux de l'Information et de la Communication, Grenoble, GRESEC, Université Stendhal Grenoble 3: http://lesenjeux.u-grenoble3. $\mathrm{fr} / 2010$-supplementB/Chaudironlhadjadene/index.html.

Ingwersen P. (1996). "Cognitive perspectives of information retrieval interaction: Elements of a cognitive IR theory ». In Journal of Documentation, vol.52, pp.3-50.

Ingwersen P. and Järvelin K. (2005). The Turn: Integration of Information Seeking and Retrieval in Context, Dordrecht: Springer, Springer e-books, 2005, 450 p.

IsaacH., CampoyE. et Kalika M. (2007). «Surcharge informationnelle, urgence et TIC. L'effet temporel des technologies de l'information ". In Management \& Avenir, vol.3, n¹3, pp.149-168. 
Johnson D. J. (2003). «On contexts of information seeking ». In Information Processing and Management, vol.39, n5, pp.735-760.

Krikelas J. (1983). «Information Seeking Behavior: Patterns And Concepts». In Drexel Library Quarterly, n², pp.5-20.

Kulthau C. (1991). "Inside the Search Process: Information Seeking from the User's Perspective». In Journal of The American Society for Information Science, vol.42, n5, pp.361-371.

Lahire B. (1996). «La variation des contextes en sciences sociales: remarques épistémologiques ». In Annales. Histoire, Sciences Sociales, ${ }^{51}{ }^{\mathrm{e}}$ Année, $\mathrm{n}^{\circ} 2$, mars-avril, pp.381-407.

Lahire B. (2001). L'homme pluriel: les ressorts de l'action, Paris, Nathan, $271 \mathrm{p}$.

Leckie G. J., Pettigrew K.E. et Sylvain C. (1996). "Modeling the information seeking of professionals: A general model derived from research on engineers, health care professionals, and lawyers ". In Library Quarterly, vol.66, n², pp.161-193.

Miège B. (2004). L'information-communication, objet de connaissance, Bruxelles, De Boeck, 248 p.

Miège B. (2007). La société conquise par la communication, Les Tic entre innovation technique et ancrage social, tome3, Grenoble, Presses Universitaires de Grenoble, $235 \mathrm{p}$.
MizzaroS. (1997). «Relevance: the whole history ». In Journal of the American Society for Information Science, vol. 48, n 9, pp.810-832.

Mucchielli A. (2006). Etudes des communications: le dialogue avec la technologie, Armand Colin, 256 p.

Munisk N. (2012). La gestion de l'information en contexte-Enquête sur les pratiques informationnelles des ingénieurs-chercheurs d'EDF $R \& D$, thèse de doctorat en Sciences de l'information et de la communication, CNAM, novembre.

PaganelliC. et MounierE. (2009). «Stratégies informationnelles en milieu professionnel: du réseau personnel à la Toile». In Congrès international ACSI (association canadienne des sciences de l'information). Tracer l'horizon informationnel du $X X{ }^{e}$ siècle: Frontières, passerelles et carrefours, 28-30 mai2009, Ottawa, 18 p.: http://www. cais-acsi.ca/proceedings/2009/ Paganelli_Mounier_2009.pdf.

PaganelliC. (2012). "Analyse des discours sur la notion d"usage' dans deux revues en sciences de I'information: Doc-SI et BBF ». In Documentaliste-Sciences de I'Information, vol.49, n², pp.64-71.

PaganelliC. (2013). "Les activités informationnelles en contexte professionnel: questionnements en information-communication ». In Clavier V. et PaganelliC. (dir.). L'information professionnelle, Paris, Hermès Sciences et Lavoisier, pp.221-240. 
Pettigrew K., Fidel R. et Bruce H. (2001). «Conceptual frameworks in information behavior ". In Annual Review of Information Science and Technology, n³5, pp.43-78.

Raynaud D. (2006). «Le contexte est-il un concept pertinent de l'explication sociologique?». L'Année sociologique, Presses Universitaires de France, 56, pp.309-330.

Rosenbaum H. (1996). "Structure and action: Towards a new concept of the information use environment ", in ASIS'96 Annual Conference Proceeding, 19-24Octobre.

Saracevic T. (1997). «The stratified model of information retrieval interaction: Extension and applications ". In Proceedings of the American Society for Information Science, vol.34, pp.313-327.

Savolainen R. (2004). «Enthusiastic, realistic and critical. Discourses of Internet use in the context of everyday life information seeking ". In Information Research, vol.10, n¹, paper198: http://InformationR. net/ir/10-1/paper198.html.

SimonnotB. (2002). «De la pertinence à l'utilité: le cas du web». In Couzinet V. et Regimbeau G. (dir.). Recherches récentes en sciences de l'information: convergences et dynamiques, Paris, ADBS Éditions, pp.393-410.

Schmoll P. (1996). Contexte(s), Revue Scolia, $n^{\circ} 6,256 \mathrm{p}$.

Staii A. (2013). «Les formes de
I'information professionnelle: esquisse des mutations structurelles d'une médiation élargie». In Clavier V. et Paganellic. (dir.). L'information professionnelle, Paris, Hermès Science, Lavoisier, pp.27-46.

Suchman L. (1987). Plans and situated actions: the problem of human/ machine communication, Cambridge, Cambridge University Press.

Tamura S., Miwa M., Saito Y., Koshizuka M., KasaiY., Matsubayashi M. et Ikeya N. (2007). «Information sharing between different groups: a qualitative study of information service to business in Japanese public libraries". In Information Research, vol.12, n²: http://InformationR.net/ir/12-2/paper306.html.

TaylorR.S. (1991). «Information use environments ». In BrendaD. (dir.), Progress in Communication Sciences, Norwood, NJ: Ablex, vol.10, pp. 217-225.

ThivantE. (2005). «Information seeking and use behaviour of economists and business analysts ". In Information Research, $n^{\circ} 10$, vol.4, $19 \mathrm{p}$.

Wilson T.D. (1981). «On user studies and information needs». In Journal of Documentation, 1981, vol.37, n¹, pp. 3-15: http://informationr.net/tdw/ publ/papers/1981infoneeds.html.

Wilson T.D. (1999). «Models in information behavior research ». In Journal of Documentation, $n^{\circ} 55$, pp. 249-270.

XieH. (2007). «Shifts in information-seeking strategies in 
information retrieval in the digital age: Planned-situational model». In Information Research, vol.12, $\mathrm{n}^{\circ} 4$ : http://InformationR.net/ ir/12-4/colis/colis22.html. 
